\title{
Control of HVAC Systems via Scenario-based Explicit MPC
}

\author{
Alessandra Parisio, Luca Fabietti, Marco Molinari, Damiano Varagnolo, Karl H. Johansson
}

\begin{abstract}
Improving energy efficiency of Heating, Ventilation and Air Conditioning (HVAC) systems is a primary objective for the society. Model Predictive Control (MPC) techniques for HVAC systems have recently received particular attention, since they can naturally account for several factors, such as weather and occupancy forecasts, comfort ranges and actuation constraints. Developing effective MPC based control strategies for HVAC systems is nontrivial, since buildings dynamics are nonlinear and affected by various uncertainties. Further, the complexity of the MPC problem and the burden of on-line computations can lead to difficulties in integrating this scheme into a building management system.

We propose to address this computational issue by designing a scenario-based explicit MPC strategy, i.e., a controller that is simultaneously based on explicit representations of the MPC feedback law and accounts for uncertainties in the occupancy patterns and weather conditions by using the scenarios paradigm. The main advantages of this approach are the absence of a-priori assumptions on the distributions of the uncertain variables, the applicability to any type of building, and the limited on-line computational burden, enabling practical implementations on low-cost hardware platforms.

We illustrate the practical implementation of the proposed explicit MPC controller on a room of a university building, showing its effectiveness and computational tractability.
\end{abstract}

\section{INTRODUCTION}

Heating, Ventilation and Air Conditioning (HVAC) systems play a fundamental role in maintaining acceptable indoor comfort levels; reports indicate that HVAC systems in developed countries contribute for approximately one fifth of the total national energy usages [1]. Current practice shows its limits, with potential energy savings achievable by using systematic building management being estimated from 5\% to $30 \%$ of the total consumptions [2], [3]. An effective controller for HVAC systems should incorporate time-dependent energy costs, bounds on the control actions, comfort requirements, as well as account for system uncertainties, e.g., weather conditions and occupancy. A natural scheme that achieves the systematic integration of all the aforementioned elements is the Model Predictive Control (MPC) [4].

Simulations in [5], [6], as well as the experimental results on real buildings reported in [7], [8], [9], show that MPC schemes can yield better comfort levels and energy use performance than current practices.

A. Parisio, L. Fabietti, M. Molinari, and K. H. Johansson are with the ACCESS Linnaeus Centre, School of Electrical Engineering, KTH Royal Institute of Technology, SE-100 44 Stockholm, Sweden. D. Varagnolo is with the Department of Computer Science, Electrical and Space Engineering, Luleå University of Technology, Forskargatan 1, SE-971 87 Luleå, Sweden. The research leading to these results was supported by the European Institute of Technology (EIT) Information and Communication Technology (ICT) Labs, the Swedish Research Council and the Knut and Alice Wallenberg Foundation. Corresponding author: parisio@kth.se.
In particular, successful implementations are likely to be based on stochastic MPC schemes with probabilistic constraints, i.e., the so called chance constrained MPCs [10]. Indeed, current standards explicitly account for the possibility of comfort violations, stating that the probability of these violations should not exceed certain levels [11].

Literature review: there is a vast literature on stochastic MPC schemes for HVAC control, e.g., [12], [13], [14]. Nonetheless, to simplify the tractability of the MPC problem the aforementioned works restrict the disturbances to have Gaussian distributions. Furthermore, these approaches do not explicitly consider the uncertainty in the occupancy when controlling the $\mathrm{CO}_{2}$ levels.

These two issues are addressed in [15], [16], where authors consider scenario-based approximations of a chance constrained MPC problem that accounts for uncertainty by extracting the scenarios from general probability distributions, thus not restricted to be Gaussian [17].

The numerical simulations performed in these works suggest that scenarios-based techniques outperform other predictive methods.

However, all these MPC strategies come with computational demand issues, since they need to run on-line optimization algorithms to solve the MPC optimization problem. In addition, scenario-based approaches requires to generate a large number of scenarios on-line. The drawback associated to this on-line computational burden is that implementation on cheap hardware platforms (such as in Programmable Logic Controllers (PLCs)) and integration in Building Automation Systems (BASs) may become prohibitive. A possible solution is to determine explicitly, off-line, the solution of the MPC optimization problem as a function of the current building state. This leads to explicit solutions of the MPC problem, which are obtained by solving multiparametric programs [18], [19], where the state vector is treated as a vector of parameters. The optimal control profile is available as a Piecewise Affine (PWA) function of the initial state.

By doing so, the computation associated with MPC reduces to a function evaluation problem, which requires simple software and cheap hardware. Besides these practical benefits, the explicit control structure also may provide insights on the structure of the control action. However, the applicability of these methods is limited to fairly small problems because of memory requirements and off-line computational times.

The few works that provide explicit MPC control laws for building temperature control, [20], [21], show, through simulations, that the explicit MPC can outperform control logics used in the buildings industry and provide performance 
comparable to a controller assuming perfect knowledge of future disturbances.

Statement of Contributions: with respect to the state-ofthe-art, we: $i$ ) compute the explicit state-feedback solution of a Scenario-based Model Predictive Control (SMPC) for both temperature and $\mathrm{CO}_{2}$ levels control;

Outline of the manuscript: Section II present the model of the building, while Section III describes the novel controller. Section IV provides and discusses experimental results, while Section V eventually summarizes our conclusions and proposes some future directions.

\section{BUILDING Modeling}

The following thermal model is tailored for the explicit MPC paradigm, and trades-off the accuracy of the predictions with the numerical tractability of the control problem. The model is required to capture the main room temperature dynamics while keeping the number of states limited. The requirement on the size of the state is mandatory, since the off-line computational time for the computation of the MPC solution increases dramatically with the problem dimension.

We adopt a two states thermal model of a single thermal zone (or room) using the quantities defined in Table I. Extensions to whole building enclosures or to room networks are conceptually straightforward and motivated by the fact that the overall building energy use is commonly computed as the sum the energy usages of the single thermal zones [22].

\begin{tabular}{ll}
\hline$A_{\text {win }}$ & windows area \\
$C$ & heat emission (per occupant) \\
$C_{\mathrm{CO}_{2}, \mathrm{i}}$ & $\mathrm{CO}_{2}$ concentration of the air inlet \\
$C_{p, a}$ & air specific heat capacity \\
$G$ & solar heat gain coefficient \\
$g_{\mathrm{CO}_{2}}$ & $\mathrm{CO}_{2}$ emitted per occupant \\
$I$ & incident solar radiation on the windows \\
$m_{\mathrm{vent}}$ & ventilation massflow \\
$N_{p}$ & number of room occupants \\
$R_{\mathrm{ae}}$ & window thermal resistance between the indoor and the out- \\
& door temperature \\
$R_{\mathrm{aw}}$ & thermal resistance between the indoor air and the walls \\
$R_{\mathrm{we}}$ & thermal resistance between the wall and outdoor air \\
$T_{\mathrm{air}}$ & average temperature of the indoor air \\
$T_{\mathrm{aver}}$ & equivalent average temperature (accounts for heat transfer \\
& though the walls and external air temperatures) \\
$T_{\mathrm{ext}}$ & outdoor temperature \\
$T_{\mathrm{mr}}$ & mean radiant temperature of the radiators \\
$T_{\mathrm{sa}}$ & air inlet temperature \\
$T_{\mathrm{wall}}$ & average temperature of the walls \\
$\Delta T_{\mathrm{h}, \text { rad }}$ & $T_{\mathrm{mr}}-T_{\text {air }}$ \\
$\Delta T_{\mathrm{vent}}$ & $T_{\mathrm{sa}}-T_{\text {air }}$ \\
\hline
\end{tabular}

TABLE I

QUANTITIES INVOLVED IN THE BUILDING MODELS.

\section{A. Thermal and $\mathrm{CO}_{2}$ models}

We model a thermal zone as a network of two nodes, one accounting for the dynamics of the air within the zone, and the other one accounting for the dynamics of the walls. In formulas,

$$
\left\{\begin{aligned}
C_{a} \dot{T}_{\mathrm{air}}= & \frac{T_{\mathrm{wall}}-T_{\mathrm{air}}}{R_{\mathrm{aw}}}+\frac{T_{\mathrm{ext}}-T_{\mathrm{air}}}{R_{\mathrm{ae}}} \\
& +C N_{p}+G A_{\mathrm{win}} I+Q_{\mathrm{vent}}+Q_{\mathrm{heat}} \\
C_{w} \dot{T}_{\mathrm{wall}}= & \frac{T_{\mathrm{air}}-T_{\mathrm{wall}}}{R_{\mathrm{aw}}}+\frac{T_{\mathrm{aver}}-T_{\mathrm{wall}}}{R_{\mathrm{we}}}
\end{aligned}\right.
$$

where

- $Q_{\mathrm{vent}}=\dot{m}_{\mathrm{vent}} C_{\mathrm{pa}} \Delta T_{\mathrm{vent}}$ represents the heat flow due to the ventilation system;

- $Q_{\text {heat }}=A_{\text {rad }} h_{\text {rad }} \Delta T_{\mathrm{h}, \text { rad }}$ is the heating flow due to the heating system (e.g., radiators);

- $T_{\text {aver }}$ is an equivalent temperature accounting for the different temperatures of the zone walls calculated as

$$
T_{\mathrm{aver}}=\frac{\sum_{i=1}^{6} T_{w, \mathrm{out}}^{i} A^{i}}{\sum_{i=1}^{6} A^{i}}
$$

i.e., as the average of the temperatures on the external side of the $i$-th wall, $T_{w \text {,out }}$, weighted by the $i$-th wall area, $A^{i}$;

As for the dynamics of the $\mathrm{CO}_{2}$ concentration in the room, we consider the model in [23], i.e.,

$$
\dot{C}_{\mathrm{CO}_{2}}=\left(\dot{m}_{\mathrm{vent}}^{\mathrm{CO}_{2}} C_{\mathrm{CO}_{2}, \mathrm{i}}+g_{\mathrm{CO}_{2}} N_{p}\right)-\dot{m}_{\mathrm{vent}} C_{\mathrm{CO}_{2}}
$$

where the inlet air concentration $C_{\mathrm{CO}_{2}, \mathrm{i}}$ is assumed equal to outdoor $\mathrm{CO}_{2}$ concentration levels.

\section{B. Control-Oriented Modeling}

Nonlinearities in the dynamics (1a) and (3) can lead to intractable multiparametric optimization problems. To address this issue, we derive linear reformulations of (1a) and (3), which are then discretized with opportune sampling time $\Delta T$. In the next paragraphs we will describe the obtained state-space linear models of $\mathrm{CO}_{2}$ concentration and room temperature levels.

State-space model for the $\mathrm{CO}_{2}$ dynamics: we consider the discrete-time Linear Time Invariant (LTI) system

$$
\begin{aligned}
x_{\mathrm{CO}_{2}}(k+1) & =a x_{\mathrm{CO}_{2}}(k)+b u_{\mathrm{CO}_{2}}(k)+e w_{\mathrm{CO}_{2}}(k) \\
y_{\mathrm{CO}_{2}}(k) & =x_{\mathrm{CO}_{2}}(k)
\end{aligned}
$$

where:

- $x_{\mathrm{CO}_{2}}$, i.e., the state, is equal to $C_{\mathrm{CO}_{2}}-C_{\mathrm{CO}_{2}, \mathrm{i}}$;

- $w_{\mathrm{CO}_{2}}$, i.e., the disturbance, is equal to the number of occupants $N_{p}$;

- $u_{\mathrm{CO}_{2}}$, i.e., the control input, is defined as $\dot{m}_{\mathrm{vent}}^{\mathrm{CO}_{2}} \cdot x_{\mathrm{CO}_{2}}$, hence it hides the bilinear term in (3). Once $u_{\mathrm{CO}_{2}}(k)$ is computed and $x_{\mathrm{CO}_{2}}(k)$ is known, one derives immediately $\dot{m}_{\mathrm{vent}}^{\mathrm{CO}_{2}}(k)$ by a simple inversion. Notice that, to meet the physical bounds on the original control input $\dot{m}_{\text {vent }}^{\mathrm{CO}_{2}}(k), u_{\mathrm{CO}_{2}}(k)$ must satisfy

$$
\dot{m}_{\mathrm{vent}}^{\min } \cdot x_{\mathrm{CO}_{2}}(k) \leq u_{\mathrm{CO}_{2}}(k) \leq \dot{m}_{\mathrm{vent}}^{\max } \cdot x_{\mathrm{CO}_{2}}(k) .
$$

State-space model for the thermal dynamics: we consider the discrete-time linear system

$$
\begin{aligned}
x_{\mathrm{T}}(k+1) & =A_{\mathrm{T}} x_{\mathrm{T}}(k)+B_{\mathrm{T}}(k) u_{\mathrm{T}}(k)+E_{\mathrm{T}} w_{\mathrm{T}}(k) \\
y_{\mathrm{T}}(k) & =C_{\mathrm{T}} x_{\mathrm{T}}(k)
\end{aligned}
$$

where

- $x_{\mathrm{T}}(k)$, i.e., the state, is a vector containing the temperatures of the indoor air and the walls;

- $w_{\mathrm{T}}(k)$, i.e., the disturbance, is a vector containing the outdoor temperature, the solar radiation and internal 
heat gains are due to occupancy, equipments and lightings;

- $y_{\mathrm{T}}(k)$, i.e., the output, is the measured indoor temperature at time $k$;

- $u_{\mathrm{T}}(k)$, i.e., the control input, is defined as $\left[\Delta T_{h}(k), \Delta T_{c}(k), \Delta u_{h}(k), \Delta u_{c}(k), \Delta T_{h, \mathrm{rad}}(k)\right]$.

The input matrix $B_{\mathrm{T}}(k)$ is time varying since it depends on $\dot{m}_{\mathrm{vent}}^{\mathrm{CO}_{2}}(k)$, as illustrated in the following.

In the thermal model (1), the control inputs are the temperature of the supplied air, $T_{\mathrm{sa}}$, the mean radiant temperature of the radiators, $T_{\mathrm{mr}}$, and the massflow $\dot{m}_{\text {vent }}$. These inputs allow to control two different heat flows, $Q_{\text {vent }}=\dot{m}_{\mathrm{vent}} C_{\mathrm{pa}}\left(T_{\mathrm{sa}}-\right.$ $\left.T_{\text {air }}\right)$ and $Q_{\text {heat }}=A_{\text {rad }} h_{\text {rad }} \Delta T_{\mathrm{h}, \text { rad }}$. We assume the convention that $Q_{\text {vent }}$ is positive when the system requires heating, and negative when it requires cooling.

Notice then the presence of the bilinear term $\dot{m}_{\text {vent }} \Delta T_{\text {vent }}$ in $Q_{\text {vent }}$. Notice moreover that $\dot{m}_{\text {vent }}$ must be at least equal to $\dot{m}_{\text {vent }}^{\mathrm{CO}_{2}}$, the latter representing the minimum massflow needed to maintain optimal $\mathrm{CO}_{2}$ levels.

To hide the bilinear term above and simultaneously account for the minimum requirements on the massflow levels we introduce the nonnegative variables $\Delta T_{h}, \Delta T_{c}, \Delta u_{h}$ and $\Delta u_{c}$ such that

$$
\begin{aligned}
\Delta T_{h}-\Delta T_{c} & =T_{\mathrm{sa}}-T_{\mathrm{air}} \\
\Delta u_{h}-\Delta u_{c} & =\Delta \dot{m}_{\mathrm{vent}} \Delta T_{\mathrm{vent}},
\end{aligned}
$$

where $\Delta \dot{m}_{\text {vent }}:=\dot{m}_{\text {vent }}-\dot{m}_{\text {vent }}^{\mathrm{CO}_{2}}$ represents the additional massflow required for guaranteeing the thermal comfort. Summarizing, the controllable inputs for (6) are

$$
u_{\mathrm{T}}(k)=\left[\begin{array}{llll}
\Delta T_{h}(k) \Delta T_{c}(k) \Delta u_{h}(k) \Delta u_{c}(k) \Delta T_{h, \operatorname{rad}}(k)
\end{array}\right] .
$$

With these new variables $Q_{\text {vent }}$ can be written as

$$
Q_{\mathrm{vent}}=\dot{m}_{\mathrm{vent}}^{\mathrm{CO}_{2}} C_{\mathrm{pa}}\left(\Delta T_{h}-\Delta T_{c}\right)+C_{\mathrm{pa}}\left(\Delta u_{h}-\Delta u_{c}\right) .
$$

Consider then that the control objective aims to minimize the thermal energy use, that may be required for either cooling or heating the space. Hence, instead of considering $Q_{\text {vent }}$, that can be either positive or negative, we need to model in the objective function of the MPC optimization problem its absolute value $\left|Q_{\text {vent }}\right|$, that can then be written as

$$
\left|Q_{\text {vent }}\right|=\dot{m}_{\text {vent }}^{\mathrm{CO}_{2}} C_{\mathrm{pa}}\left(\Delta T_{h}+\Delta T_{c}\right)+C_{\mathrm{pa}}\left(\Delta u_{h}+\Delta u_{c}\right) .
$$

Once $\dot{m}_{\text {vent }}^{\mathrm{CO}_{2}}(k)$ and $u_{\mathrm{T}}(k)$ have been computed, $T_{\mathrm{sa}}(k)$, $T_{\mathrm{mr}}(k)$ and $\dot{m}_{\text {vent }}(k)$ can be easily computed by simple inversions. Consider also that the input-to-state matrix $B_{\mathrm{T}}(k)$ is time varying, since it accounts for the time-varying $\dot{m}_{\text {vent }}^{\mathrm{CO}_{2}}(k)$.

Eventually we can account for the physical bounds on the original control inputs at each time step $k$ by accounting for the bounds

$$
\left\{\begin{array}{l}
T_{\mathrm{sa}}^{\mathrm{min}}-T_{\mathrm{air}}(k) \leq \Delta T_{h}(k)-\Delta T_{c}(k) \leq T_{\mathrm{sa}}^{\max }-T_{\mathrm{air}}(k)(7 \mathrm{a}) \\
\Delta u_{h}(k) \leq \Delta \dot{m}_{\mathrm{vent}}^{\max }(k) \Delta T_{h}(k) \\
\Delta u_{c}(k) \leq \Delta \dot{m}_{\mathrm{vent}}^{\max }(k) \Delta T_{c}(k)
\end{array}\right.
$$

where $\Delta \dot{m}_{\mathrm{vent}}^{\max }(k):=\dot{m}_{\mathrm{vent}}^{\max }-\dot{m}_{\mathrm{vent}}^{\mathrm{CO}_{2}}(k)$.

\section{SCENARIO-BASED EXPLICIT MPC FOR HVAC SYSTEMS}

We detail the proposed control scheme with Section IIIA, which describes the overall architecture and outlines the algorithm for the on-line implementation of the explicit statefeedback control law, and with Section III-B, which then provides the control objectives and formulate the corresponding MPC problem.

\section{A. Control Architecture and On-line Implementation}

Figure 1 depicts the architecture of the implemented control system. The indoor temperature and air $\mathrm{CO}_{2}$ levels are measured and then controlled through the ventilation system and radiators. These devices are actuated using low-level Proportional Integrative (PI) controllers, which setpoints are computed by means of our explicit Explicit Scenario-based Model Predictive Control (ESMPC) scheme.

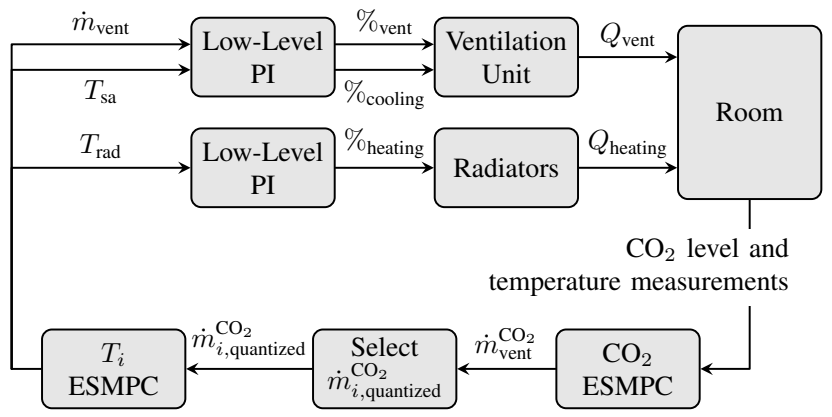

Fig. 1. Architecture of the control system implemented on the testbed.

To improve the computational tractability of the overall control problem, we take advantage of the independence of the $\mathrm{CO}_{2}$ dynamics from the thermal ones and the priority of $\mathrm{CO}_{2}$ comfort. This allows us to consider two separated multiparametric subproblems, discussed in Section III-B: $i$ ) the $\mathrm{CO}_{2}$-ESMPC problem (minimize the energy used to keep $\mathrm{CO}_{2}$ levels in given comfort bounds); $i$ ) the T-ESMPC problem (minimize energy use to keep temperature levels in given comfort bounds while preserving the minimum massflow computed by the $\mathrm{CO}_{2}$-ESMPC controller).

By solving the $\mathrm{CO}_{2}$-ESMPC and the T-ESMPC problems we obtain explicit state-feedback laws that are expressed as PWA function of the state $x$ [18], [19]. Hence, the $\mathrm{CO}_{2}$ ESMPC and T-ESMPC control laws have respectively the form

$$
\begin{aligned}
u^{E \mathrm{CO}_{2}}(x) & =Q_{i}^{E \mathrm{CO}_{2}} x+q_{i}^{E \mathrm{CO}_{2}} & & \text { if } H_{i}^{E \mathrm{CO}_{2}} x \leq K_{i}^{E \mathrm{CO}_{2}} \\
u^{E T}(x) & =Q_{j}^{E T} x+q_{j}^{E T} & & \text { if } H_{j}^{E T} x \leq K_{j}^{E T}
\end{aligned}
$$

where the polyhedral sets $\mathcal{X}_{i}^{E \mathrm{EO}_{2}}:=\left\{H_{i}^{E \mathrm{CO}_{2}} x \leq K_{i}^{E \mathrm{CO}_{2}}\right\}$ and $\mathcal{X}_{j}^{E T}:=\left\{H_{j}^{E T} x \leq K_{j}^{E T}\right\}$, with $j=1 \ldots N^{E T}$ are partitions of the set of states, with $i=1, \ldots, N^{E \mathrm{CO}_{2}}$ and $j=1, \ldots, N^{E T}$.

To guarantee that the overall procedure satisfies the requirements on the minimum massflow needed to maintain comfortable $\mathrm{CO}_{2}$ levels, we proceed as follows: the set of 
admissible values of the massflow is partitioned using standard algorithms (e.g., Lloyd algorithm [24]) and $Q$ quantized values are computed, $\dot{m}_{1 \text {,quantized }}^{\mathrm{CO}_{2}}, \ldots, \dot{m}_{Q \text {,quantized }}^{\mathrm{CO}_{2}}$. Then, for each $\dot{m}_{i \text {,quantized }}^{\mathrm{CO}_{2}}$, a corresponding $\mathrm{T}_{i}$-ESMPC problem that takes $\dot{m}_{i \text {,quantized }}^{\mathrm{CO}_{2}}$ as lower bound on the massflow is solved [15].

The scenario-based controller can be then implemented on-line according to Algorithm 1.

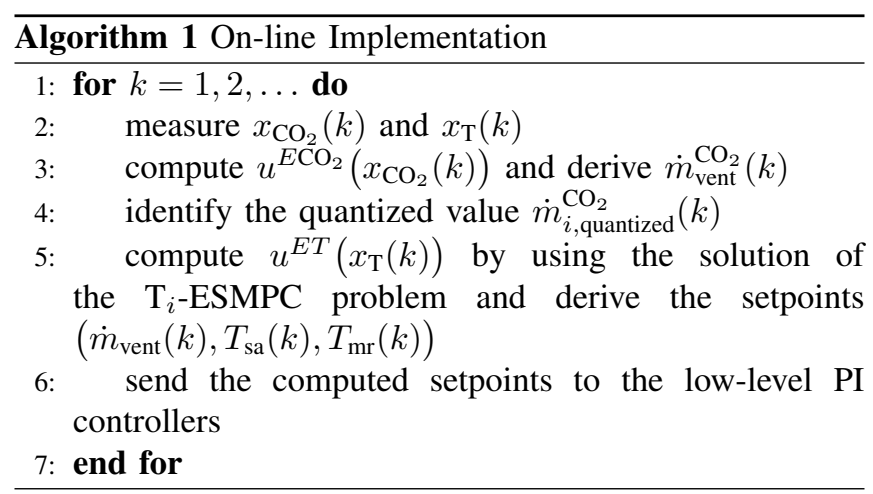

\section{B. Control Objective}

The proposed control strategy aims at optimizing HVAC operations so that the thermal energy use is minimized while indoor temperature and $\mathrm{CO}_{2}$ levels are kept in given comfort ranges.

As suggested in the section above, we formulate two problems, the $\mathrm{CO}_{2}$-ESMPC problem and the T-ESMPC problem. Since both problems consider discrete-time linear models and consider hard constraints on the inputs and probabilistic constraints on the outputs $\left(\mathrm{CO}_{2}\right.$ levels and temperature respectively), we consider an MPC problem for the control of discrete-time systems of the form

$$
\begin{aligned}
x(k+1) & =A x(k)+B(k) u(k)+E w(k) \\
y(k) & =C x(k),
\end{aligned}
$$

where $x(k) \in \mathcal{R}^{n}$ is the state, $u(k) \in \mathcal{R}^{m}$ is the control input, $w(k) \in \mathcal{R}^{r}$ is the stochastic disturbance and $y(k) \in \mathcal{R}^{p}$ is the output. Indeed (8) represents either (4) or (6), depending on the controller under consideration $\left(\mathrm{CO}_{2}\right.$ ESMPC or T-ESMPC).

At each time step $k$ we consider a prediction horizon $N$ and define

$$
\begin{aligned}
\boldsymbol{x} & :=\left[x(k+1 \mid k)^{\mathrm{T}}, \ldots, x(k+N \mid k)^{\mathrm{T}}\right]^{\mathrm{T}}, \\
\boldsymbol{u} & :=\left[u(k)^{\mathrm{T}}, \ldots, u(k+N-1)^{\mathrm{T}}\right]^{\mathrm{T}}, \\
\boldsymbol{y} & :=\left[y(k+1 \mid k)^{\mathrm{T}}, \ldots, y(k+N \mid k)^{\mathrm{T}}\right]^{\mathrm{T}}, \\
\boldsymbol{w} & :=\left[w(k)^{\mathrm{T}}, \ldots, w(k+N-1)^{\mathrm{T}}\right]^{\mathrm{T}},
\end{aligned}
$$

where $x(k+i+1 \mid k)=A x(k+i \mid k)+B u(k+i)+E w(k+1)$ and $y(k+i \mid k)=C x(k+i \mid k)$ are the $i$-steps-ahead state and output predictions. The dynamics of the predicted output can be written in compact form as a function of the initial state $x(k)$, i.e., as

$$
\boldsymbol{y}=\boldsymbol{C}_{A} x(k)+\boldsymbol{C}_{B} \boldsymbol{u}+\boldsymbol{C}_{E} \boldsymbol{w}
$$

where the prediction dynamics matrices $C_{A}, C_{B}, C_{E}$ can be easily derived from (8).

We then assume three types of constraints: $i)$ inputs constraints of the form $u^{\min } \leq F u(k) \leq u^{\max }$; constraints (7b) and $(7 \mathrm{c})$ can be expressed as input constraints; ii) comfort constraints on the outputs of the form $y^{\min } \leq y(k) \leq y^{\max }$ $\left.{ }^{1}\right)$; iii) mixed constraints on outputs and disturbances of the form $G_{y} y(k)+G_{u} u(k) \leq g$; constraints (5) and (7a) can be expressed as mixed constraints.

Given the fact that both the constraints and dynamics (9) are linear, we can compactly write constraints on the inputs, the outputs and mixed constraints as

$$
\begin{aligned}
\boldsymbol{G}_{x k} x(k)+\boldsymbol{G}_{u} \boldsymbol{u}+\boldsymbol{G}_{w} \boldsymbol{w} & \leq \boldsymbol{g} \\
\boldsymbol{F} \boldsymbol{u} & \leq \boldsymbol{f},
\end{aligned}
$$

with opportune $\boldsymbol{F} \in \mathcal{R}^{q \times m N}, \boldsymbol{f} \in \mathcal{R}^{q}, \boldsymbol{G}_{w} \in \mathcal{R}^{r \times p N}$, $\boldsymbol{G}_{u} \in \mathcal{R}^{r \times m N}, \boldsymbol{G}_{x k} \in \mathcal{R}^{r \times n}$ and $\boldsymbol{g} \in \mathcal{R}^{r}$.

We then assume the possibility of violating the comfort bounds on the indoor temperature and $\mathrm{CO}_{2}$ levels with a predefined probability, i.e., formulate output constraints as the following chance-constrains

$$
\mathbb{P}\left[\boldsymbol{G}_{x k} x(k)+\boldsymbol{G}_{u} \boldsymbol{u}+\boldsymbol{G}_{w} \boldsymbol{w} \leq \boldsymbol{g}\right] \geq 1-\alpha
$$

with $\alpha \in[0,1]$ being the violation probability level. In these formulations $\alpha$ represents a tradeoff between performance and constraint satisfaction.

As mentioned above, our control strategy aims at minimizing the thermal energy use. This means that the cost function in the proposed MPC problem formulations is the energy use over the whole prediction horizon,e.g., $\sum_{k=0}^{N-1}\left(\left|Q_{\text {vent }}\right|+\right.$ $\left.Q_{\text {heat }}\right)$ in the T-ESMPC problem. Denoting by $\boldsymbol{c}^{\mathrm{T}} \boldsymbol{u} \Delta k, \boldsymbol{c} \in$ $\mathbb{R}^{m N}$, the cost vector for both the T-ESMPC and the $\mathrm{CO}_{2}$ ESMPC and $\Delta k$ the sampling period, the general control problem can be formally stated as

$$
\begin{array}{ll}
\min _{\boldsymbol{u}} & \boldsymbol{c}^{\mathrm{T}} \boldsymbol{u} \Delta k \\
\text { s.t. } & \mathbb{P}\left[\boldsymbol{G}_{x k} x(k)+\boldsymbol{G}_{u} \boldsymbol{u}+\boldsymbol{G}_{w} \boldsymbol{w} \leq \boldsymbol{g}\right] \geq 1-\alpha \\
& \boldsymbol{F} \boldsymbol{u} \leq \boldsymbol{f} .
\end{array}
$$

Chance constrained problems like (11) are generally intractable unless the uncertainties follow specific distributions, e.g., Gaussian [10].

Since Gaussian assumptions are rather restrictive, specially to handle strongly heteroskedastic processes like occupancy patterns, we propose to overcome this limitation and obtain a solvable MPC problem by applying randomized approaches [25], [26]. These approaches do not require to specify the probability distribution of the uncertainties, but just the possibility to extract i.i.d. samples, also called scenarios, from their distribution. In [25], [27] the minimum number of samples guaranteeing that considering constraints (??) will lead to a feasible solution for Problem (11) with a given confidence level is provided. Recently the scenario approach has been applied in an MPC framework [28], where authors have shown that bounds on the number of scenarios

\footnotetext{
${ }^{1}$ We incidentally notice that comfort bounds are usually time-varying.
} 
can be improved if the problem contains multiple chance constraints (e.g. MPC problems), or if a chance constraint belongs to a special class of constraints (e.g. linear or quadratic constraints). In these cases the conservativeness of the scenario solution can be reduced. Moreover, the authors in [29] show that the theory can be extended to the case where a given number of sampled constraints are discarded a posteriori, so that the objective value achieved by the scenario solution can be improved.

Hence, as in [9], we model the uncertainty in occupancy levels, outdoor temperatures, and incident solar radiation as independent stochastic processes and estimate their probability distributions from historical data. From these learned distributions, we generate a set of $S$ i.i.d. scenarios, $\boldsymbol{w}_{1}, \ldots, \boldsymbol{w}_{S}$, where $\boldsymbol{w}_{i}:=\left[w_{i}^{\mathrm{T}}(0), \ldots, w_{i}^{\mathrm{T}}(N-1)\right]^{\mathrm{T}}, i=$ $1, \ldots, S$. Then the chance-constrained problem (11) can be approximated with the following deterministic problem

$$
\begin{array}{ll}
\min _{\boldsymbol{u}} & \boldsymbol{c}^{\mathrm{T}} \boldsymbol{u} \Delta k \\
\text { s.t. } & \boldsymbol{G}_{x k} x(k)+\boldsymbol{G}_{u} \boldsymbol{u} \leq \boldsymbol{g}-\max _{i=1, \ldots S} \boldsymbol{G}_{w} \boldsymbol{w}_{i} \\
& \boldsymbol{F} \boldsymbol{u} \leq \boldsymbol{f},
\end{array}
$$

where the max operator is applied element-wise to $\boldsymbol{G}_{w} \boldsymbol{w}_{i}$.

Problem (12) can be treated as a multiparametric linear problem by considering the vector $x(k)$ as a vector of parameters [18], [19]. Once the multiparametric problem has been solved off line, assuming that a full measurement of the state $x(k)$ is available at the current time $k$, the explicit solution is available as a piecewise affine function of $x(k)$

$$
u(k)=Q_{i} x(k)+q_{i} \quad \text { if } \quad H_{i} x(k) \leq K_{i}
$$

where the polyhedral sets $\mathcal{X}_{i}:=\left\{H_{i} x \leq K_{i}\right\}$, with $i=$ $1 \ldots N$ are a state space partition.

The parameters of the PWA function in (13), $Q_{i}, q_{i}$ and the polyhedral sets $\mathcal{X}_{i}$, can be computing by using the MPT toolbox [30], where a parametric programming solver is implemented.

We remark that the constraints in Problem (12) can be softened to guarantee that the problem with sampled constraints is always feasible.

\section{EXPERIMENTAL RESULTS}

\section{A. Experimental Setup}

We consider a laboratory room of approximatively $80 \mathrm{~m}^{2}$ in the ground floor of the Q-building of the Royal Institute of Technology (KTH) campus in Stockholm. The room has a concrete heavyweight structure with limited glass surface and one external wall, facing South-East, which is partially shaded by a parking lot. As summarized in Figure 2, its HVAC system is composed of two parts: the ventilation system, supplying fresh air, and a radiator heating system.

Fresh air is supplied by a central balanced ventilation system that operates only between 7:00 and 16:00 during work days. The ventilation system pre-conditions fresh air from outside and distributes it at a temperature of about 20$21^{\circ} \mathrm{C}$. Part of this generated air flow is then conveyed directly into the room, while part can be further cooled by a cooling

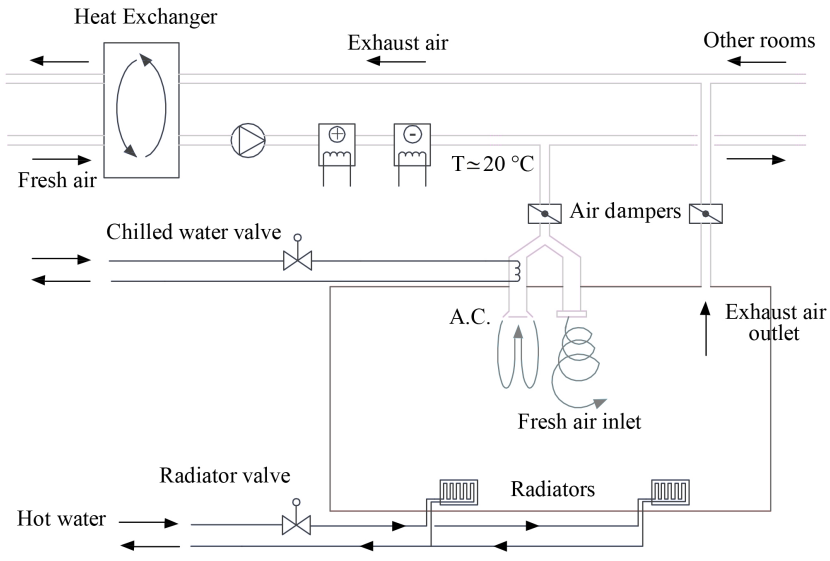

Fig. 2. Scheme of the HVAC system of the testbed.

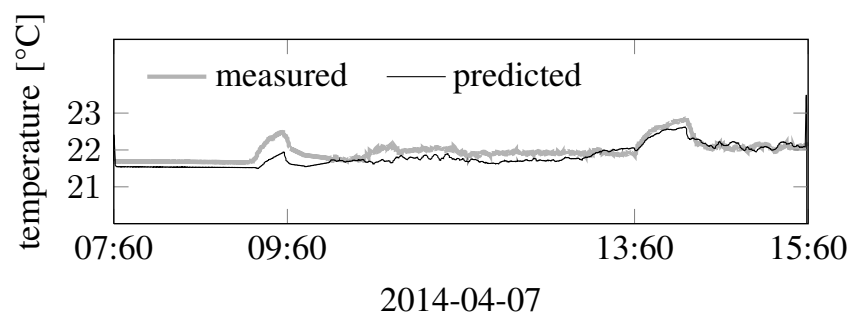

Fig. 3. Validation of the air temperature model (1a) against measured data.

coil. The controllable actuators of the ventilation system are three: two dampers, that regulate the opening of the inflow and outflow ducts, and a valve, that regulates the temperature of the air chilling circuit. When the central fan is on, a minimum level of the air flow rate is supplied independently of the occupancy level in the room.

The heating system uses instead radiators as final units. The hot water circulating in them is provided by a district heating system and has a temperature that is determined by the external temperature conditions. The unique controllable actuator is the valve regulating the flow of the hot water.

Figure 3 validates the thermal model (1a) by comparing measured and simulated indoor temperature driven by the same disturbances and the same control inputs for a time horizon of 9 hours (which is the prediction horizon considered in the ESMPC problem), starting from the same measured initial condition. The model appears capable of capturing the thermal dynamics in the room with an acceptable accuracy. Indeed, the difference between the measured and the estimated indoor temperature is always smaller than $1{ }^{\circ} \mathrm{C}$. Moreover seven days have been simulated, resulting in similar performance and an average Root-Mean-Square Error of Prediction (RMSEP) equal to 2.19.

The model can be further improved by analyzing the measured and simulated indoor temperature under different weather and occupancy profiles. 


\section{B. Evaluation of Experimental Results}

We here compare two different controllers $i$ ) the current practice, a simple control logic with PI control loops and switching logic, indicated by the acronym "AHC" (from Akademiska Hus, the company managing the building of the testbed); ii) our ESMPC.

For the latter we consider a prediction horizon of 9 hours and a sampling time of 10 minutes, hence $N=54$. The optimal control laws are computed with Matlab on an Intel(R) Core(TM)i7-2600 CPU, $3.40 \mathrm{GHz}$ and $8 \mathrm{~GB}$ of RAM. It takes 20 minutes on average to compute a optimal control law as a PWA function consisting of 800 polyhedra in the set of admissible initial conditions. After computing all the required state feedback control laws (according the control architecture 1), the profiles of the $\mathrm{CO}_{2}$ levels, the indoor temperature and the control inputs are obtained by applying Algorithm 1.

We aim at testing the performance of our ESMPC in terms of energy use and comfort as well as the effect of a different number of scenarios in real experiments. The controllers are tested on three consecutive days in March 2014, 18-20, for 9 hours each day, from 7:00 to 16:00. The comfort range of the indoor temperature has been set between $20{ }^{\circ} \mathrm{C}$ and 22 ${ }^{\circ} \mathrm{C}$.

Figure 4 shows then the experimental results. We run the AHC on the 19th, the ESMPC with 10 scenarios on the 18th and the ESMPC with 1000 scenarios on the 20th. Then we compare the AHC with the ESMPC with 10 scenarios in Test 1 and with the ESMPC with 1000 scenarios in Test 2.

Each column of Figure 4 refers to one of the tests above, while each row depicts the disturbances (i.e., outdoor temperature and occupancy), the control inputs (i.e., supply air temperature and massflow) and the controlled indoor temperature and $\mathrm{CO}_{2}$ levels. The horizontal axis reports the time period of the experiments, from 7:00 to 16:00. Despite the cold season, both controllers in both tests require cooling when there is people in the room. The demand for cooling during the cold season can be explained by the relatively high internal gains, due to occupancy and equipment, and by the limited and moderately insulated external walls surface. This implies that the thermal indoor dynamics are significantly affected by the occupancy patterns.

Notice that on the 18th and on the 20th, the days when the ESMPC was applied, both the outdoor temperature and the occupancy was higher compared to the day when AHC was employed. In particular, the occupancy was significantly higher on the 20th, after lunch time. Despite these more challenging disturbances, the temperature profiles resulting from the ESMPC are very similar to the ones obtained by AHC. The ESMPC is capable to keep the temperature profile close to the upper bound, although the ESMPC with 10 scenarios violated this bound more often.

Despite the temperature profiles resulting form the two controllers being indeed close, the ESMPC achieved this result at a significant lower energy cost. This is a promising performance, especially considering the higher occupancy

and outdoor temperatures that the ESMPC needed to compensate. This can be clearly seen by looking at the required massflow in Figure 4: the AHC turns on/off the cooling system quite often, while the ESMPC uses the cooling system fewer times, i.e., only when really convenient. E.g., consider Test 2 at 14:30, and notice how there is a peak in the massflow that tries to compensate the increased outdoor temperature and the upcoming peak in the occupancy ( 8 people).

Test-1
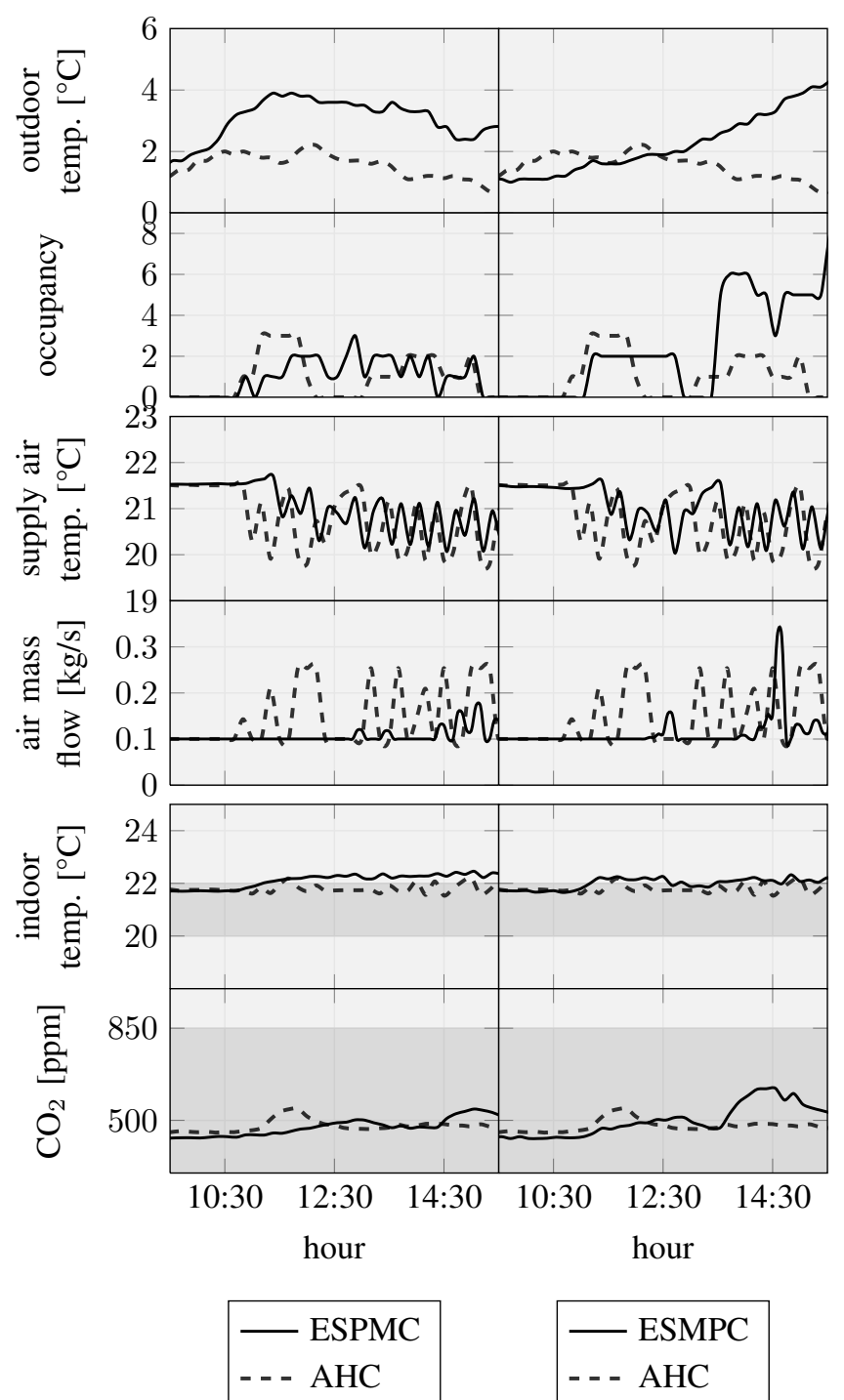

Fig. 4. Disturbances, $\mathrm{CO}_{2}$ levels, indoor temperatures and control inputs profiles for high- and low-occupancy experimental tests. The shaded areas represent the comfort bounds.

We consider the total energy usage as performance index, calculated as $E_{\text {tot }}=c_{\text {pa }} \sum_{k=0}^{N-1} \dot{m}_{\text {vent }}(k) \mid T_{\mathrm{sa}}(k)-$ $T_{\text {room }}(k) \mid \Delta k[\mathrm{kWh}]$. Numerically, for the ESMPC $E_{\text {tot }}=$ $0.77 \mathrm{kWh}$ on the $20 \mathrm{th}$, and $E_{\mathrm{tot}}=0.79 \mathrm{kWh}$ on the $18 \mathrm{th}$, while for the $\mathrm{AHC} E_{\mathrm{tot}}=1.04 \mathrm{kWh}$, approximatively $31-33 \%$ higher. 


\section{Conclusions and Future Studies}

In this work we propose an Explicit Scenario-based Model Predictive Control (ESMPC) controller for Heating, Ventilation and Air Conditioning (HVAC) systems, aiming to diminish the energy required to maintain indoor thermal comfort and acceptable $\mathrm{CO}_{2}$ levels.

To account for the probabilistic nature of the disturbances affecting the building, we endowed the Model Predictive Control (MPC) scheme with a learning module that infers the statistics of the disturbances from the data without requiring any Gaussian assumption for the statistics of the disturbances. Since implicit MPCs schemes requires solving on-line optimization problems and dedicated hardware, it can be prohibitive to integrate them into a building management system. To cope with these problems we developed explicit versions of the previously implemented strategies, which lead to controllers with simpler hardware and software requirements.

Our explicit approach is, in our opinion, more advanced and complete than the explicit MPC controllers for HVAC schemes currently existing in literature. Indeed, we both consider dynamics of $\mathrm{CO}_{2}$ and temperature, and (at the same time) consider a scenario-based approach, that accounts for the uncertainties on the forecasts of the disturbances.

The strategy has then been implemented and tested on a real room of a university building, showing that the resulting actuation laws can be more effective than the current practice.

The promising results achieved in real experimentations motivate efforts to improve the method, in particular by applying the procedure and the results illustrated in [29], [28]

A further important future work is towards the generalization of the control scheme to the case of whole buildings, which leads to increased modeling effort. Another relevant direction is to investigate how it is possible to adapt the Piecewise Affine (PWA) control laws when the statistics of the disturbances are updated.

\section{REFERENCES}

[1] European Commission, "Debate Europe-building on the experience of plan D for democracy, dialogue and debate," European Economic and Social Committee and the Committee of the Regions, COM 158/4, Brussels, 2008.

[2] A. Costa, M. M. Keane, J. I. Torrens, and E. Corry, "Building operation and energy performance: Monitoring, analysis and optimisation toolkit," Applied Energy, vol. 101, pp. 310-316, Jan. 2013.

[3] K. Chua, S. Chou, W. Yang, and J. Yan, "Achieving better energy-efficient air conditioning - A review of technologies and strategies," Applied Energy, vol. 104, pp. 87-104, Apr. 2013.

[4] M. Morari, J. Lee, and C. Garcia, Model Predictive Control. Prentice Hall, 2001.

[5] J. Hua and P. Karavab, "Model Predictive Control Strategies for Buildings with Mixed-Mode Cooling," Building and Environment, vol. 71, pp. 233-244, 2014.

[6] M. Fadzli Haniff, H. Selamat, R. Yusof, S. Buyamin, and F. Sham Ismail, "Review of HVAC scheduling techniques for buildings towards energy-efficient and cost-effective operations," Renewable and Sustainable Energy Reviews, vol. 27, pp. 94-103, Nov. 2013.

[7] D. Sturzenegger, D. Gyalistras, M. Gwerder, C. Sagerschnig, M. Morari, and R. S. Smith, "Model Predictive Control of a Swiss office building," in Clima-RHEVA World Congress, 2013.
[8] J. Široký, F. Oldewurtel, J. Cigler, and S. Prívara, "Experimental analysis of model predictive control for an energy efficient building heating system," Applied Energy, vol. 88, no. 9, pp. 3079-3087, Sept. 2011.

[9] A. Parisio, D. Varagnolo, D. Risberg, G. Pattarello, M. Molinari, and K. H. Johansson, "Randomized Model Predictive Control for HVAC Systems," in Proceedings of the 5th ACM Workshop on Embedded Systems For Energy-Efficient Buildings, 2013.

[10] P. Kall and J. Mayer, Stochastic Linear Programming: Models, Theory, and Computation. Springer-Verlag, 2005.

[11] BSI, "En 15251:2007: Indoor environmental input parameters for design and assessment of energy performance of buildings addressing indoor air quality, thermal environment, lighting and acoustics," British Standards Institute, Tech. Rep., 2008.

[12] Y. Ma and F. Borrelli, "Fast stochastic predictive control for building temperature regulation," in American Control Conference, 2012, pp. 3075-3080.

[13] Y. Ma, S. Vichik, and F. Borrelli, "Fast stochastic MPC with optimal risk allocation applied to building control systems," in IEEE Conference on Decision and Control. Ieee, Dec. 2012, pp. 7559-7564.

[14] F. Oldewurtel, A. Parisio, C. N. Jones, D. Gyalistras, M. Gwerder, V. Stauch, B. Lehmann, and M. Morari, "Use of model predictive control and weather forecasts for energy efficient building climate control," Energy and Buildings, vol. 45, no. 45, pp. 15-27, Feb. 2012.

[15] A. Parisio, D. Varagnolo, G. Pattarello, L. Fabietti, and K. H. Johansson, "Implementation of a Scenario-based MPC for HVAC Systems: an Experimental Case Study," in 19th World Congress of the International Federation of Automatic Control, to appear, 2014.

[16] X. Zhang, G. Schildbach, D. Sturzenegger, and M. Morari, "Scenario-Based MPC for Energy-Efficient Building Climate Control under Weather and Occupancy Uncertainty," in European Control Conference (ECC), 2013, pp. 1029-1034.

[17] G. C. Calafiore and L. Fagiano, "Robust Model Predictive Control via Scenario Optimization," IEEE Transactions on Automatic Control, vol. 58, no. 1, pp. 219-224, 2013.

[18] A. Alessio and A. Bemporad, A Survey on Explicit Model Predictive Control, nonlinear ed., L. Magni, D. Raimondo, and F. Allgöwer, Eds. Berlin Heidelberg: Springer-Verlag, 2009

[19] A. Bemporad, F. Borrelli, and M. Morari, "Model predictive control based on linear programming the explicit solution," Automatic Control Laboratory, ETH, Zurich, Switzerland, Tech. Rep., 2002.

[20] J. Drgona, M. Kvasnica, M. Klauco, and M. Fikar, "Explicit Stochastic MPC Approach to Building Temperature Control," in 2013 IEEE 52nd IEEE C onference on Decision and Control (CDC), 2013.

[21] S. Koehler and F. Borrelli, "Building Temperature Distributed Control via Explicit MPC and Trim and Respond Methods," European Control Conference (ECC), pp. 4334-4339, 2013.

[22] M. Gwerder and J. Toedtli, "Predictive control for integrated room automation," in Clima - RHEVA World Congress, 2005.

[23] H. A. Aglan, "Predictive model for $\mathrm{CO}_{2}$ generation and decay in building envelopes," Journal of Applied Physics, vol. 93, no. 2, pp. $1287-1290,2003$.

[24] M. J. Sabin and R. Gray, "Global convergence and empirical consistency of the generalized Lloyd algorithm," Information Theory, IEEE Transactions on, vol. 32, no. 2, pp. 148-155, Mar 1986.

[25] G. Calafiore, "Random Convex programs," SIAM Journal on Optimization, vol. 20, no. 6, pp. 3427-3464, 2010.

[26] G. Schildbach, G. C. Calafiore, L. Fagiano, and M. Morari, "Randomized Model Predictive Control for Stochastic Linear Systems," in American Control Conference, 2012.

[27] G. Calafiore and M. Campi, "The Scenario Approach to Robust Control Design," IEEE Transactions on Automatic Control, vol. 51, no. 5, pp. 742-753, 2008.

[28] G. Schildbach, L. Fagiano, C. Frei, and M. Morari, "The Scenario Approach for Stochastic Model Predictive Control with Bounds on Closed-Loop Constraint Violations," pp. 1-18, July 2013.

[29] M. C. Campi and S. Garatti, "A Sampling-and-Discarding Approach to Chance-Constrained Optimization: Feasibility and Optimality," Journal of Optimization Theory and Applications, vol. 148, no. 2, pp. 257-280, 2011

[30] M. Kvasnica, P. Grieder, M. Baotic, and M. Morari, "Multi-parametric toolbox (MPT)," in Hybrid Systems: Computation and Control, ser. Lecture Notes in Computer Science, R. Alur and G. Pappas, Eds. Springer Berlin Heidelberg, 2004, vol. 2993, pp. 448-462. 